OPEN ACCESS

Edited by:

Y. K. O. Teng,

Leiden University Medical

Center, Netherlands

Reviewed by:

Stephen McAdoo,

Imperial College London,

United Kingdom

Xavier Bossuyt,

KU Leuven, Belgium

*Correspondence:

Vladimir Tesar

vladimir.tesar@vfn.cz

Specialty section:

This article was submitted to Autoimmune and Autoinflammatory Disorders,

a section of the journal

Frontiers in Immunology

Received: 10 August 2020

Accepted: 22 February 2021

Published: 22 March 2021

Citation:

Tesar V and Hruskova Z (2021)

Autoantibodies in the Diagnosis,

Monitoring, and Treatment of

Membranous Nephropathy.

Front. Immunol. 12:593288

doi: 10.3389/fimmu.2021.593288

\section{Autoantibodies in the Diagnosis, Monitoring, and Treatment of Membranous Nephropathy}

\author{
Vladimir Tesar* and Zdenka Hruskova \\ Department of Nephrology, 1st Faculty of Medicine, General University Hospital, Charles University, Prague, Czechia
}

The discovery of anti-podocyte antibodies in primary membranous nephropathy (MN) has revolutionized our approach toward the diagnosis and treatment of this disease. Evaluation of serum levels of anti-podocyte antibodies paved the way for non-invasive diagnosis and helped distinguish between primary and secondary MN although the relationship between anti-podocyte antibodies and cancer remains to be elucidated. Serum levels of anti-PLA2R antibodies directed against the major podocyte autoantigen are related to $\mathrm{MN}$ activity and the decrease in serum levels of anti-PLA2R antibodies in response to treatment (immunologic remission) also serves as an early indicator of the later putative proteinuric remission, enabling personalization of the treatment. The serum levels of anti-podocyte antibodies also enable the prediction of renal outcomes in terms of both remission and the risk of progression to end-stage renal disease. The positivity of anti-PLA2R antibodies before renal transplantation is associated with the risk of recurrence of $\mathrm{MN}$. It remains to be established if all these relations observed in patients with anti-PLA2R antibodies are also valid for expanding spectrum of antibodies directed against recently discovered minor antigens (e.g., THSD7A, NELL-1, semaphorin 3B).

Keywords: membranous nephropathy, anti-PLA2R antibodies, anti-THSD7A antibody, remission, outcome

\section{MEMBRANOUS NEPHROPATHY-A MAJOR CAUSE OF NEPHROTIC SYNDROME}

Membranous nephropathy (MN) is defined by the typical histopathological patterns of thickening of the glomerular capillary wall with subepithelial immune deposits gradually embedded in newly formed glomerular basement membranes with positive granular immunofluorescence of IgG and C3 $(1,2)$. Although kidney biopsy is an invasive procedure, it remains the gold standard for the diagnosis of $\mathrm{MN}$.

More than $80 \%$ of patients with $\mathrm{MN}$ present with nephrotic syndrome (3). MN is the cause of nephrotic syndrome in approximately $25 \%$ of adults (more frequently in males) and is the most common cause of nephrotic syndrome among older adults (4). The glomerular filtration rate at presentation is usually normal or only slightly impaired.

MN may be secondary, most often as one of the histologic classes (class V) of lupus nephritis, or pathogenetically related to cancer, hepatitis $B$, sarcoidosis, or some drugs $(1,2)$. However, in about $75 \%$ of patients with $\mathrm{MN}$, the primary cause is not apparent, with the disease traditionally classified as idiopathic (until the discovery of anti-podocyte antibodies) or primary MN. Light microscopy does not reliably distinguish between primary and secondary MN. Immunofluorescent deposition 
of IgG4 is typical for primary and IgG1, IgG2, and IgG3 for secondary $\mathrm{MN}$ with $\mathrm{C} 1 \mathrm{q}$ often present in membranous (class $\mathrm{V}$ ) lupus nephritis $(1,2)$.

Proteinuria and serum creatinine are still used to stratify the renal risk in patients with primary $\mathrm{MN}(5,6)$. Patients with sub-nephrotic proteinuria have good long-term renal outcomes and should not be treated with immunosuppression. On the other hand, predicting renal outcomes in patients with nephrotic proteinuria is much more difficult as it ranges from spontaneous remission [in as much as $50 \%$ of patients during longer follow-up (7)] to the progression to end-stage kidney disease [in about $30 \%$ of patients within 10 years of follow-up (8)].

Due to the high propensity to spontaneous remission and toxicity of traditional treatment according to KDIGO guidelines (9) immunosuppressive treatment with alkylating agents is initiated only in patients with nephrotic syndrome persisting for at least 6 months, severe complications of nephrotic syndrome, or an increase in serum creatinine by $30 \%$ within $6-12$ months.

\section{EXPANDING SPECTRUM OF ANTI-PODOCYTE ANTIBODIES IN MN}

The experimental model of MN, Heymann nephritis (10), was induced in its active form by the immunization of the rats with the material derived from the proximal tubule and in its passive form by injecting the rats with heterologous IgG to a crude rat tubular extract (11).

Heymann nephritis and human $\mathrm{MN}$ were always suspected to be autoimmune diseases putatively caused by anti-podocyte antibodies. As a proof of the concept in 1995, megalin, expressed by rat podocytes and on the luminal membrane of the cells of the proximal tubules (where it is involved in the uptake of a variety of proteins) was identified as an autoantigen of Heymann nephritis (12).

In humans, megalin is believed to be expressed only by the brush border of the proximal tubule, but not the human podocytes. Therefore, it could not be the target antigen in human MN. Recently, however, primary renal interstitial disease with anti-brush border antibodies and IgG-positive immune deposits along the tubular basement membrane, segmental subepithelial glomerular deposits, subnephrotic proteinuria, and circulating antibodies against megalin (LDL receptor-related protein 2LRP2) was described $(13,14)$. Segmental MN in humans with anti-megalin antibodies is apparently due to some (although not as dense as in rats) expression of megalin in human podocytes (14). However, megalin is not the podocyte autoantigen in patients with typical MN.

The role of podocyte proteins in eliciting an immune response in $\mathrm{MN}$ was first demonstrated with the finding of neonatal MN in a child of a mother with a mutated MME gene for neutral endopeptidase (NEP), normally expressed by podocytes. During pregnancy, the mother raised antibodies against NEP of the fetus, which crossed the placenta and caused transient $\mathrm{MN}$ in the newborn (15).

However, the major podocyte autoantigen in MN was only identified in 2009 as the phospholipase A2 receptor [PLA2R
(16)]. Anti-PLA2R antibodies occur with disease presentation in about $70-80 \%$ of patients (more often men). The presence of antiPLA2R antibodies is highly specific for primary MN. However, they may also be present in some patients with secondary MN (sarcoidosis or HBV-associated $\mathrm{MN}$ ).

Since 2009, several minor podocyte autoantigens have been identified in patients with $\mathrm{MN}$ : antibodies against thrombospondin type-1 domain-containing 7A [THSD7A (17)] are present in $3-5 \%$ of patients (more often women) (6), antibodies against neural epidermal growth factor-like 1 protein [NELL-1 (18)] are present in $5-10 \%$ of patients, and antibodies against semaphorin 3B (19) are present namely in pediatric patients. Anti-NELL-1 antibodies were shown to be much more common in segmental MN (20). Very recently, antibodies against protocadherin 7 (PCDH7) and high termperature recombinant protein A1 /HTRA1), both of IgG4 subclass, were described in several patients with anti-PLA2R-negative primary membranous nephropathy $(21,22)$.

Additionally, antibodies directed against exostosin-1 and exostosin-2 (EXT-1 and EXT-2) were found to be specific for lupus $\mathrm{MN}$ (23) and antibodies against neural cell adhesion molecule-1 (NCAM-1) occur in about $6 \%$ of patients with lupus $\mathrm{MN}$, but may also occur in $2 \%$ of patients with primary MN (24). In membranous lupus nephritis EXT1/EXT2-positive patients have compared to EXT1/EXT2-negative patients more often nephrotic proteinuria, but less glomerulosclerosis and interstitial fibrosis. EXT1/EXT2-negative patients progressed to end-stage kidney disese more quickly and more frequently (25).

Antibodies to EXT1/EXT2, NELL-1 and Sema3B are of IgG1 subtype and are complement-activating, antibodies to PCDH7 are, similarly as anti-PLA2R antibodies predominantly of IgG4 subtype and do not activate the complement.

Different autoantibodies may thus be associated with different pathogenesis, pattern of histologic damage and different clinical phenotype including response to treatment and outcome (26).

All as-yet identified autoantigens are responsible for up to $90 \%$ of cases of idiopathic (primary) $\mathrm{MN}$, so the future discovery of further minor antigens cannot be excluded (27). Approximately $1 \%$ of patients with $\mathrm{MN}$ may have double positivity for both anti-PLA2R and anti-THSD7A antibodies $(28,29)$. In a large cohort of 1,012 patients with biopsy-proven MN, anti-THSD7A antibodies were identified in $2.8 \%$ of patients, eight of which $(0.8 \%)$ were double positive for anti-THSD7A and anti-PLA2R antibodies (30).

Double positivity of MPO-ANCA and anti-PLA2R was described in two patients with ANCA-associated glomerulonephritis combined with MN-like lesions (31). In other studies necrotizing/crescentic glomerulonephritis was described in a small proportion (about $0,3 \%$ ) of patients with membranous nephropathy $(32,33)$. Although most of these patients have concomitant ANCA or anti-GBM antibodies this histologic pattern was observed also in several patients with anti-PLA2R antibodies only. It remains unclear if anti-GBM damage caused by ANCA or anti-GBM antibodies may expose podocyte antigens, or, on the other hand, subepithelial deposits in membranous nephropathy may enhance damage to GBM by ANCA or anti-GBM antibodies. 


\section{GLOMERULAR BINDING OF ANTI-PLA2R ANTIBODIES}

The binding of anti-PLA2R IgG4 antibodies to podocytes can usually be detected in patients with $\mathrm{MN}$ using immunofluorescence or immunohistochemistry. However, it is not always parallel to circulating anti-PLA2R antibodies, if measured at the time of biopsy $(34,35)$. Although glomerular binding of anti-PLA2R antibodies is highly specific for primary $\mathrm{MN}$, it also occurs in some patients with HBV, sarcoidosis, or cancer-associated secondary membranous nephropathies.

Glomerular binding of anti-PLA2R antibodies may persist for months even after the systemic autoimmune response is suppressed. Alternatively, the absence of circulating anti-PLA2R antibodies is observed in some patients with active $\mathrm{MN}$ and glomerular anti-PLA2R binding. It is speculated that the lack of circulating antibody detection could be due to the high avidity of anti-PLA2R antibodies to the podocytes (35).

In some patients with $\mathrm{MN}$ positive for glomerular PLA2R staining, circulating antibodies may not be detectable at presentation, but may occur later during follow-up (and treatment) of the disease (36). This seroconversion could be explained by the buffering capacity of the kidney, that is, circulating antibodies occur only after the binding capacity of the kidney is exceeded, emphasizing the importance of the evaluation of glomerular PLA2R staining or repeated evaluation of antiPLA2R in anti-PLA2R negative patients, especially during the first 6-months of follow-up.

In some patients with positive glomerular staining, circulating anti-PLA2R antibodies may only become detectable at 12 months, or even during late relapse at 28 months (37). There can be even an early absence of glomerular IgG4-antiPLA2R positive staining, as some patients may have initially IgG1 dominant immune deposits (potentially with another podocyte target antigen), that are PLA2R negative. This suggests putative later histologic conversion (to IgG4-PLA2R staining) which could be demonstrated only through repeat kidney biopsies (38). Positive glomerular staining of anti-THSD7A (39) and anti-NELL-1 antibodies (18) was also described in patients with $\mathrm{MN}$ with autoantibodies directed to these minor autoantigens, respectively.

\section{ANTI-PODOCYTE ANTIBODIES IN THE PATHOGENESIS OF MN}

The role of complement in the pathogenesis of $\mathrm{MN}$ was demonstrated by the deposition of C3 and C5b-9 in both Heymann nephritis and human MN. Moreover, glomerular injury in Heymann nephritis can be prevented by blocking the formation of the C5b-9 complex on the podocyte membrane (40).

Although the direct pathogenic role of anti-podocyte autoantibodies in MN has been supported by the observation in passive Heymann nephritis induced in rats by injection of IgG against tubular extract (11), in humans, it was difficult to reconcile the preponderance of non-complement activating IgG4 anti-PLA2R antibodies (at least at a later course of anti-PLA2R antibody-positive MN) with the welldocumented complement activation. One putative explanation could be the activation of the alternative complement pathway in $\mathrm{MN}$ recently demonstrated using proteomic analysis of microdissected glomeruli (41), which may be preceded by earlier glomerular deposition of other IgG subclasses that activated the classical complement pathway (35). The recently described antiNELL-1 antibody is predominantly of the IgG1 class and maybe the cause of prominent C3 deposits (40). The role of complement regulatory proteins in the pathogenesis of $\mathrm{MN}$ also remains to be elucidated (42).

THSD7A (unlike PLA2R1) is expressed in human and murine podocytes. As a result, it was possible to show that administration of human anti-THSD7A antibodies to mice results in their binding to murine podocytes with subsequent foot process effacement, histologic changes typical of MN and proteinuria (43). However, in this model, proteinuria preceding complement deposition was only small and transient (44).

The pathogenic role of anti-PLA2R antibodies was recently demonstrated in a mouse model in which the major target antigen, human PLA2R, was expressed in mouse podocytes in vivo (45). In this model the administration of heterologous rabbit anti-PLA2R antibodies (with no data on IgG subclass) increased dose-dependently proteinuria (to the nephrotic range) with limited C3 deposition, leaving the proportional role of IgG4 predominant anti-PLA2R antibodies and membrane attack complex (C5b-9) unclear. It is conceivable that the early rise of proteinuria in experimental models of MN may be complementindependent as both anti-THSD7A and anti-PLA2R antibodies were shown to directly mediate podocyte damage in $\mathrm{MN}$. However, their interaction with complement activation (if any) remains to be elucidated.

There is currently ongoing trial in anti-PLA2R antibodypositive primary membranous nephropathy with an oral inhibitor of factor B iptacopan (LNP023) directed of the alternative complement pathway (NCT04154787) and one small phase 2 safety trial with anti-MASP2 monoclonal antibody narsoplimab (NCT02682407) inhibiting complement lectin pathway.

Epitope spreading may play an important role in the severity of podocyte injury. Commonly in autoimmune diseases, primary response to immunodominant antigens may expand to another epitope on the same protein or to dominant epitopes on neighboring molecules $(6,46)$. It has been shown $(47)$ that antiPLA2R antibodies may be restricted only to the cysteine-rich (CysR) domain, or the anti-PLA2R response may also spread the C-type lectin domain 1 (CTLD1) and C-type lectin domain 7 (CTLD7) of PLA2R. Epitope spreading was shown to be associated with a more severe course of the disease.

\section{GENETICS OF MN AND ANTI-PODOCYTE ANTIBODIES}

An early GWAS in 556 European patients (48) identified two loci strongly associated with MN: the PLA2R1 gene on chromosome 2q24 and HLA-DQA1 on chromosome 6p21 with homozygosity 
for both alleles, increasing the risk of MN more than 78 times. A similar kind of association (allele in the HLA system likely facilitating the response against the target antigen and the risk allele of the target antigen itself) has also been described for PR3-positive ANCA-associated vasculitis (49). The strong association of three single nucleotide polymorphisms (SNPs) within PLA2R1 and one within HLA-DQA1 was confirmed in 1,112 Chinese patients with MN (50). Risk alleles were associated with the presence of anti-PLA2R antibodies and their glomerular expression. Retrospective analysis of anti-PLA2R antibodies and genotyping of DQ alleles and analysis of PLA2R1 SNPs performed in 90 prevalent patients with idiopathic MN with a 90-month follow-up demonstrated that levels of antiPLA2R antibodies were significantly linked to DQA1*05:01 and DQB1*02:01 (51).

A recent large GWAS in 3782 patients of European and East Asian ancestry (52) identified two other risk loci (NFKB1 and IRF4) and provided a fine mapping of PLA2R1 (rs178312151). Interestingly, this GWAS demonstrated the ancestry-specific effect of HLA alleles: DQA1*0501 in Europeans, DRB1*1501 in East Asians, and DRB1*0301 in both ethnicities with identified loci explaining the $32 \%$ disease risk in East Asians and 25\% in Europeans. All these studies clearly showed that $\mathrm{MN}$ and more specifically an autoimmune response to PLA2R are strongly genetically mediated. The genetic contribution of the identified risk loci is much stronger in $\mathrm{MN}$ compared with IgA nephropathy, where it explains no more than $5 \%$ of the overall disease risk (53).

\section{ANTI-PODOCYTE ANTIBODIES AND NON-INVASIVE DIAGNOSIS OF MN}

As anti-podocyte antibodies are highly specific for primary $\mathrm{MN}$ [well-documented for anti-PLA2R antibodies, where the specificity is $98-100 \%$ (54)] it has been suggested that the diagnosis of primary $\mathrm{MN}$ may be assessed in patients with nephrotic syndrome, normal renal function and positive antiPLA2R antibodies even without renal biopsy (54). However, there are several drawbacks. Anti-PLA2R antibodies should be measured with ELISA, and the reference ranges should be better established (55). Limited data are available concerning antiPLA2R antibody specificity in non-white populations. Larger studies are required to help to define the positive predictive value of anti-PLA2R positivity as this testing is usually performed in selected patient populations with and without nephrotic syndrome and, anti-PLA2R positivity was shown to precede clinical diagnosis of $\mathrm{MN}$ by months to years (56). In these patients, however, anti-PLA2R positivity could predict the future development of nephrotic syndrome. As expected, increasing sensitivity can be achieved only at the expense of decreasing specificity $[<75 \%$ with a low cutoff level (57)].

The specificity of anti-PLA2R and anti-THSD7A antibodies is very high in terms of comparing patients with $\mathrm{MN}$ and other glomerular diseases. Both autoantibodies are also more frequent in primary $\mathrm{MN}$ (58); however, specificity with which anti-PLA2R positivity excludes secondary $\mathrm{MN}$, especially cancer-associated
$\mathrm{MN}$ is lower. Similarly, anti-THSD7A antibodies are highly specific for $\mathrm{MN}$; however, they also do not reliably distinguish between primary and secondary MN (39).

Of note, anti-PLA2R positivity cannot be used as a noninvasive diagnostic test in patients with impaired renal function and also, in patients with suspected concomitant diabetic kidney disease or other glomerular diseases. Renal biopsies also provide an estimate of the degree of tubulointerstitial damage, strongly associated with renal outcomes and may also have a substantial impact on the response to immunosuppressive treatment. Systemic disease, although rare, namely sarcoidosis or antineutrophil cytoplasmic antibody (ANCA)-associated vasculitis, should also be considered in patients with antiPLA2R positive antibodies. Rare necrotizing/crescentic glomerulonephritis in $\mathrm{MN}$ canot be also recognized without renal biopsy $(32,33)$.

\section{ANTI-PLA2R AND ANTI-THSD7A ANTIBODIES AND CANCER}

According to a recent meta-analysis (59), the prevalence of cancer in $\mathrm{MN}$ is $10 \%$ (most commonly lung cancer in $26 \%$ of patients, followed by prostate cancer in $15 \%$ of patients) with a diagnosis of cancer preceding the diagnosis of $\mathrm{MN}$ in $20 \%$ of patients.

THSD7A is expressed by some cancers and it has been suggested that anti-THSD7A antibodies are associated with an increased risk of cancer-associated MN (60), and screening for cancer was recommended in patients with anti-THSD7A positive $\mathrm{MN}$ (61).

In a large Chinese study, anti-THSD7A antibodies were positive in only $2 \%$ of patients with cancer-associated $\mathrm{MN}$ (29). Surprisingly, $41 \%$ of patients with cancer-associated MN had anti-PLA2R1 antibodies. In another Chinese study (62), $33 \%$ of patients with cancer-associated $\mathrm{MN}$ were positive for both circulating anti-PLA2R antibodies and glomerular (IgG4-dominant) PLA2R staining. Although 56\% of patients had THSD7A positive staining in cancer tissues, circulating antibodies were not detected. These studies suggest that antiPLA2R antibodies may be quite commonly (and anti-THSD7A uncommonly) positive in (at least Chinese) patients with cancerassociated MN. However, it is unclear whether these findings are purely coincident, or if PLA2R antibodies participate in the pathogenesis of cancer-associated $\mathrm{MN}$.

In a recent study in Caucasian patients (63), anti-PLA2R antibodies were positive in $75 \%$ of patients with idiopathic $\mathrm{MN}$ and in 9 out of 32 (28\%, in seven patients with cancer) with secondary MN. The specificity of anti-PLA2R antibodies decreased from 100 and $94.6 \%$ compared to normal and pathological (other glomerulonephritides) controls to only $71.9 \%$ vs. secondary $\mathrm{MN}$.

Antigen-specific IgG subclasses and their association with cancer were studied in patients with anti-PLA2R antibodies or anti-THSD7A antibodies (64). All patients had the most frequent IgG4 antibodies. In patients with idiopathic MN, IgG subclass distribution levels of IgG4 antibodies were similar to those of patients with malignancy-associated MN. This suggested that the 
pathogenesis of primary and malignancy-associated $\mathrm{MN}$ with either anti-PLA2R or anti-THSD7A antibodies may be similar to those in primary $\mathrm{MN}$.

In the consecutive series of anti-PLA2R and anti-THSD7A negative patients $3.8 \%$ had NELL-1 as a target antigen with segmental or incomplete IgG staining dominantly of IgG1 subclass. Out of them $33 \%$ had concurrent malignancy which is higher proportion than in anti-PLA2R and anti-THSD7A positive patients (65).

Although cancer is more common in anti-PLA2R antibodynegative patients, diagnostic workup for cancer cannot be completely avoided, even in patients with anti-PLA2R positive antibodies (66). Elucidation of mechanisms underlying anti-podocyte autoimmunity induced by cancer warrants further research.

\section{ANTI-PODOCYTE ANTIBODIES AND THE OUTCOME OF MN}

Up to a third of patients with MN may develop spontaneous remission and can be spared immunosuppressive treatments, sometimes associated with serious adverse events. This is reflected by the recommendation that immunosuppressive treatment should be postponed by as many as 6 months in patients without poor prognostic signs, such as impaired glomerular filtration rate and high-grade proteinuria (9).

The chance of developing spontaneous remission was shown to be related to the titer of anti-PLA2R antibodies (67-70) and is comparatively low in patients with high titers of anti-PLA2R antibodies who could be treated with immunosuppression without unnecessary delay.

Spontaneous remission occurred significantly less often in patients with high antibody titers [38 vs. $4 \%$ in the lowest and highest tertiles (68)]. Patients with low antiPLA2R levels had 2.72 times higher rates of spontaneous remission after a median follow-up of 2.9 years. Alternatively, high anti-PLA2R levels were associated with persistent proteinuria and a need for immunosuppressive therapy (70). Complete spontaneous remission was also more common in patients with lower anti-PLA2R levels at presentation (71).

The titer of anti-PLA2R antibodies was also closely linked to the outcome in terms of a higher risk of declining renal function during a follow-up period of 90 months (51). In another prospective study in patients with $\mathrm{MN}$ and positivity for anti-PLA2R antibodies with a median follow-up of 27 months, clinical endpoint (defined as an increase in serum creatinine by $\geq 25 \%$ and serum creatinine reaching $\geq 1.3 \mathrm{mg} / \mathrm{dL}$ ) was reached in $69 \%$ of patients in the highest tertile of the titer of anti-PLA2R antibodies compared to only $25 \%$ of patients in the lowest tertile at inclusion into study. The study endpoints were also significantly shorter in patients with the highest compared to the lowest tertile of anti-PLA2R antibodies [17.7 vs. 30.9 months (72)]. In another study, however, long-term renal survival (at 5, 10, and 15 years) was high in all tertiles of anti-PLA2R antibodies (97, 93, and $89 \%$, respectively), and was only related to the severity of proteinuria (70).

Poor renal outcomes in patients with anti-PLA2R antibodies may also be associated with epitope spreading of anti-PLA2R autoimmune response with less frequent nephrotic syndrome at presentation, a higher rate of spontaneous remission, and lower risk of progression to renal failure in patients with antiPLA2R antibodies restricted to cysteine-rich domain of the PLA2R, compared with patients with concomitant positivity of antibodies to C-type lectin domain 1 and C-type lectin domain 7 (47). Epitope spreading is strongly correlated with titers of anti-PLA2R antibodies and a lower rate of remission at 6 months (73).

Epitope spreading is thus an important independent predictor of poor outcomes, and patients with epitope spreading should be treated without delay with higher doses of rituximab. As epitope-specific assays for anti-PLA2R are not available, epitope spreading cannot be evaluated in clinical practice and we must rely on the correlation of epitope spreading with anti-PLA2R titers (73). Similar to patients with high titers of anti-PLA2R antibodies, patients with high titer of anti-THSD7A antibodies also have poor clinical outcomes (30).

In summary, low baseline and decreasing anti-PLA2R antibody levels strongly predict spontaneous remission, thus favoring conservative therapy. Conversely, high baseline or increasing anti-PLA2R antibody levels are associated with nephrotic syndrome and progressive loss of kidney function, thereby encouraging prompt initiation of immunosuppressive therapy (58).

\section{ANTIBODIES AND TRANSPLANTATION}

Up to $40-50 \%$ of patients with primary $\mathrm{MN}$ (if untreated) may finally develop end-stage renal disease. MN may recur in transplant in $30-45 \%$ of patients with recurrence being more frequently reported by centers performing regular protocol biopsies (74). Anti-PLA2R antibodies are detected in $70-80 \%$ of patients with recurrence of $\mathrm{MN}$ in the renal allograft (75). The risk of recurrence is higher in patients with positive anti-PLA2R antibodies before transplantation $(76,77)$ and with anti-PLA2R antibody staining in renal allografts (77).

Using cutoff levels of circulating anti-PLA2R antibodies of 45 $\mathrm{U} / \mathrm{mL}$, the recurrence of $\mathrm{MN}$ was predicted with a sensitivity and specificity of $85 \%$ and a negative predictive value of $92 \%$. Anti-PLA2R levels and recurrence of $\mathrm{MN}$ in the allograft were shown to be genetically mediated [associated with HLA DQA1* 05:01/05 and DQB1* 02:01 (77)]. In another study (78), antiPLA2R antibodies were positive prior to transplantation in $83 \%$ of patients who experienced recurrence.

In the patient with anti-THSD7A-associated MN, who reached end-stage renal failure and was subsequently transplanted with early recurrence of $\mathrm{MN}$ in the graft, the anti-THSD7A antibodies were detectable both before and after transplantation. The staining for THSD7A was also documented 
in the allograft, suggesting the pathogenic role of anti-THSD7A antibodies (43).

\section{ANTI-PODOCYTE ANTIBODIES AND ACTIVITY OF MN}

Titers of anti-PLA2R antibodies are strongly correlated with the clinical activity of the disease and proteinuria $(67,68,79)$. This is demonstrated by the antibody titers in patients with active disease compared with patients with partial or complete remission (51). The reemergence of circulating antibodies predicts disease relapse $(58,80)$.

Titers of anti-THSD7A also decrease (or the antibody completely disappears) with the development of remission and once again reappear (increase) with the relapse of the disease (29). High titers of anti-THSD7A are also correlated with the activity of the disease. However, due to the disease sparsity, the evidence is less convincing than that of the PLA2R antibodies (30). Regular measurement of the levels of anti-PLA2R and antiTHSD7A antibodies may thus be used for monitoring the activity of the disease.

\section{ANTI-PODOCYTE ANTIBODIES AND RESPONSE TO TREATMENT}

Patients with primary MN are currently treated with either alkylating cytotoxic drugs, calcineurin inhibitors, B-cell depleting monoclonal antibody, or rituximab. Alkylating cytotoxic drugs are effective, but their use is associated with significant adverse events. The use of calcineurin inhibitors is associated with a high relapse rate on their tapering and withdrawal.

Reduction in the levels of circulating anti-PLA2R antibodies preceded by several months and predicted a decrease in the proteinuria in rituximab-treated patients with $\mathrm{MN}(68,81-83)$.

In 133 adult patients with anti-PLA2R positive $\mathrm{MN}$, the antibody levels decreased after 3 months of immunosuppressive treatment by $81 \%$ and proteinuria by $39 \%$ (72). Patients who developed remission 12 months later had significantly lower levels of anti-PLA2R at baseline compared to patients with no remission. Moreover, patients with high levels of anti-PLA2R antibodies achieved remission later than patients with low levels. The antibody levels remained elevated in patients who did not achieve remission of proteinuria.

In another study, 84 out of 132 rituximab-treated patients with idiopathic MN achieved complete or partial remission during a median follow-up of 30.8 months and 25 relapsed after remission (80). In 81 patients with positive anti-PLA2R antibodies, remission was strongly predicted by a lower antibody titer prior to treatment. These patients had complete antibody depletion 6 months after the initiation of rituximab treatment. In all patients, depletion of anti-PLA2R antibodies preceded complete remission. Early reduction of the titer of anti-PLA2R antibody by $50 \%$ was associated with a decrease in proteinuria by $50 \%$ by 10 months. In this study, response to rituximab treatment was not related to the polymorphisms of both PLA2R1 and HLA-DQA1.
The role of immunologic remission (depletion of antiPLA2R antibodies) in MN was recently demonstrated in three randomized controlled trials, GEMRITUX (84), MENTOR (85) and STARMEN (86).

In the GEMRITUX trial (84), 75 patients with biopsyproven $\mathrm{MN}$ and nephrotic syndrome were randomized either to rituximab or non-immunosuppressive antiproteinuric treatment. Anti-PLA2R depletion was achieved at 6 months in $50 \%$ of patients treated with rituximab treated and only in $12 \%$ of patients treated with non-immunosuppressive antiproteinuric treatment. Although at 6 months there was no significant difference between both limbs in combined complete and partial remission ( 35.1 vs. $21.1 \%$ ), at the end of observational phase (after median follow-up of 17 months) remission rates were significantly higher in patients treated with rituximab compared with patients treated with non-immunosuppressive antiproteinuric treatment (64.9 vs. $34.2 \%$ ). Early responses in terms of anti-PLA2R levels predicted the later development of clinical remission.

In the MENTOR trial (85), 130 patients were randomized to rituximab or cyclosporine. Early response (at 12 months) to either rituximab of cyclosporine was similar (complete and partial remission induced in $60 \%$ vs. $52 \%$ of patients, respectively). However, at 24 months, complete or partial remission was much more frequent in rituximab- than in cyclosporine-treated patients (60 vs. $20 \%$ of patients, respectively). It should be, however, stressed, that cyclosporin was withdrawn already at 12 months, so these data reflect namely the risk of early relapse after cyclosporine withdrawal. Importantly, the decline of anti-PLA2R antibodies was faster and of greater magnitude and duration in rituximab vs. cyclosporine. This again demonstrated that immunological remission (depletion of antiPLA2R antibodies) was indispensable for clinical remission.

In a recently published STARMEN trial (86) which randomized 86 pts with membranous nephropathy at high risk or progression to either cyclical alternating treatment with corticosteroids and cyclophosphamide or tacrolimus followed by single dose of rituximab complete or partial remission of nephrotic syndrome at 24 months was reached in $83.7 \%$ of patients treated with corticosteroid-cyclophosphamide and in only $58.1 \%$ of patients treated with tacrolimus and rituximab. Immunological remission (depletion of anti-PLA2R antibodies) was achieved at 3 and 6 months in 77 and 92\%, respectively, of pts in corticosteroid-cyclophosphamide limb compared to 45 and $70 \%$ of patients in the tacrolimus-rituximab limb-in fact, it reflects the efficacy of tacrolimus only as rituximab was given only at 6 months). Corticosteroid-cyclophophamide treatment thus induced compared to tacrolimus-rituximab more often both complete and partial remission and induced imunological remission more quickly.

In a small open-label study belimumab (monoclonal antibody against B lymphocyte stimulating factor BAFF-BLyS) decrease of proteinuria was preceded by the decrease of the titer of antiPLA2R antibodies (87). There is currently ongoing clinical trial (REBOOT, NCT03949855) in anti-PLA2R positive membranous nephropathy comparing the combination of belimumab with rituximab with rituximab only. 
Rituximab was also shown to reverse epitope spreading in almost $60 \%$ of treated patients (88). In the GEMRITUX trial (84), epitope spreading was strongly correlated with the titer of circulating anti-PLA2R antibodies, and reversal of epitope spreading was observed in 10 out of 17 patients (58\%) with epitope spreading at baseline treated with rituximab.

Available data suggest that the early decrease in levels of anti-PLA2R antibodies is a predictor of later clinical remission, which may develop slowly within more than 1 year. As the chance to develop clinical remission is very high in patients with treatment-induced depletion of anti-PLA2R antibodies, no further immunosuppression (e.g., maintenance treatment with rituximab) may be indicated. Instead, monitoring and waiting may be a safer and more reasonable approach $(2,58,80)$.

Alternatively, a decrease in proteinuria without an immunological response (as in some patients treated with cyclosporine) should not be interpreted as real remission. There is a high risk of relapse when treatment (most frequently cyclosporine) is withdrawn. Persistence of high titer of antiPLA2R antibody is a sign of ongoing immunologic activity (and possibly ongoing podocyte damage) despite lower proteinuria. Thus, the efficacy of any immunosuppressive treatment should be assessed based on the induction of immunologic remission. The reappearance of anti-PLA2R positivity and/or an increase in the previously decreased titer of anti-PLA2R antibody is a clear sign of the impending relapse of $\mathrm{MN}$, sometimes preceding the rise of proteinuria. Serial measurements of anti-PLA2R antibodies (monthly, or at least bi-monthly) during and at the end of the cycle of immunosuppressive treatment may help personalize the treatment of MN (2).

Response to treatment in anti-PLA2R positive patients is characterized by a rapid decrease in the titer of anti-PLA2R antibodies. The response to treatment is worse in patients with high levels of anti-PLA2R antibodies before the start of treatment. Levels of anti-PLA2R antibodies are independent

\section{REFERENCES}

1. Ponticelli C, Glassock RJ. Glomerular diseases: membranous nephropathy - a modern view. Clin J Am Soc Nephrol. (2014) 9:609-16. doi: 10.2215/CJN.04160413

2. Glassock RJ. Antiphospholipase A2 receptor autoantibody guided diagnosis and treatment of membranous nephropathy. A new personalized medical approach. Clin J Am Soc Nephrol. (2014) 9:1341-3. doi: 10.2215/CJN.05880614

3. Donadio JV Jr., Torres VE, Velosa JA, Wagoner RD, Holley KE, Okamura M, et al. Idiopathic membranous nephropathy: the natural history of untreated patients. Kidney Int. (1988) 33:708-15. doi: 10.1038/ki.1988.56

4. Wasserstein AG. Membranous glomerulonephritis. J Am Soc Nephrol. (1997) 8:664-74.

5. Floege J, Barbour SJ, Cattran DC, Hogan JJ, Nachman PH, Tang SCW, et al. Management and treatment of glomerular diseases (part 1): conclusions from a Kidney Disease: Improving Global Outcomes (KDIGO) controversies conference. Kidney Int. (2019) 95:268-80. doi: 10.1016/j.kint.2018. 10.018

6. Cravedi P, Jarque M, Angeletti A, Fava A, Cantarelli C, Bestard O. Immunemonitoring diseaseactivity in primary membranous nephropathy. Front Med. (2019) 6:241. doi: 10.3389/fmed.2019.00241 predictors of response to treatment (in terms of achieving remission). Levels of anti-THSD7A antibodies are also related to the response to immunosuppressive treatment. In a subgroup of patients with serial titers, persistently elevated anti-THSD7A autoantibodies were observed in patients who wither did not respond to treatment or achieve remission (30). Hopefully, our experience will be soon extended to patients with anti-NELL-1 antibodies (18).

\section{CONCLUSIONS}

Identification of anti-podocy antibodies elucidated the pathogenesis of primary $\mathrm{MN}$ and enabled its non-invasive diagnosis. Titers of anti-podocyte antibodies predict the renal outcome and response to treatment and help personalize the immunosuppressive therapy. Persistent remission and prevention of progression to end-stage renal disease cannot be achieved without suppression of the podocyte-directed autoimmune response. With the identification of several further minor podocyte antigens classification of primary membranous nephropathy will be based on the type of autoantibody, its IgG subclass and potential to activate complement resulting possibly in the future preference of either B cell- or complement-targeted therapeutic approaches.

\section{AUTHOR CONTRIBUTIONS}

Both authors contributed equally to the review of the available literature and preparing the manuscript.

\section{FUNDING}

The paper was supported and the publication fee was paid by the research initiative of the Ministry of Health of Czech Republic RVO-VFN 64165.

7. Davison AM, Cameron IS, Kerr DN, Ogg CS, Wilkinson RW. The natural history of renal function in untreated membranous glomerulonephritis in adults. Clin Nephrol. (1984) 22:61-7.

8. Hogan SL, Muller KE, Jennette JC, Falk RJ. A review of therapeutic studies of idiopathic membranous glomerulopathy. Am J Kidney Dis. (1995) 25:86275. doi: 10.1016/0272-6386(95)90568-5

9. KDIGO clinical practice guideline for the treatment of glomerulonephritis. Kidney Int Supplements. (2012) 2:1-143. doi: 10.1038/kisup.2012.9

10. Heymann W, Hackel DB, Harwood S, Wilson SGF, Hunter JLP. Production of the nephrotic syndrome in rats by Freund' s adjuvants and rat kidney suspensions. Proc Soc Exp Biol Med. (1959) 100:660-4. doi: 10.3181/00379727-100-24736

11. Sugisaki T, Klassen J, Andres GA, Milgrom F, McCluskey RT. Passive transfer of Heymann nephritis with serum. Kidney Int. (1973) 3:6673. doi: $10.1038 / \mathrm{ki} .1973 .13$

12. Farquhar MG, Saito A, Kerjaschki D, Orlando RA. The Heymann nephritis antigenic comples: megalin (gp330) and RAP. J Am Soc Nephrol. (1995) 6:3547.

13. Larsen CP, Trivin-Avillach C, Coles P, Collins AB, Merchant M, Ma H, et al. LDL Receptor-related protein 2 (Megalin) as a target antigen in human kidney anti-brush border antibody disease. J Am Soc Nephrol. (2018) 29:64453. doi: 10.1681/ASN.2017060664 
14. Perez-Gomez MV, Sanchez-Nino MD, Ortiz A. Megalin/lipoprotein receptorrelated protein 2 autoimmunity and kidney disease. Clin Kidney J. (2020) 13:281-6. doi: 10.1093/ckj/sfz171

15. Debiec H, Nauta J, Coulet F, van der Burg M, Guigonis V, Schurmans $\mathrm{T}$, et al. Role of truncating mutations in MME gene in fetomaternal alloimunisation and antenatal glomerulopathies. Lancet. (2004) 364:12529. doi: 10.1016/S0140-6736(04)17142-0

16. Beck LH Jr., Bonegio RGB, Lambeau G, Beck DM, Powell DW, Cummins $\mathrm{TD}$, et al. M-type phospholipase A2 receptor as target antigen in idiopathic membranous nephropathy. N Engl J Med. (2009) 361:1121. doi: 10.1056/NEJMoa0810457

17. Tomas NM, Beck LH Jr., Meyer-Schwesinger C, Seitz-Polski B, Ma $\mathrm{H}$, Zahner $\mathrm{G}$, et al. Thrombospondin type-1 domain-containing 7A in idiopathic membranous nephropathy. N Engl J Med. (2014) 371:227787. doi: 10.1056/NEJMoa1409354

18. Sethi S, Debiec H, Madden B, Charlesworth MC, Morelle J, Gross LA, et al. Neural epidermal growth factor-like 1 protein (NELL-1) associated membranous nephropathy. Kidney Int. (2020) 97:162-74. doi: 10.1016/j.kint.2019.09.014

19. Sethi S, Debiec H, Madden B, Vivarelli M, Charlesworth MC, Ravindran A, et al. Semaphorin 3B-associated membranous nephropathy is a distinct type of disease predominantly present in pediatric patients. Kidney Int. (2020) 98:1253-64. doi: 10.1016/j.kint.2020.05.030

20. Kudose S, Santoriello D, Debiec H, Canetta PA, Bomback AS, Stokes MB, et al. The clinicopathologic spectrum of segmental membranous glomerulopathy. Kidney Int. (2021) 99:247-55. doi: 10.1016/j.kint.2020.06.014

21. Sethi S, Madden BJ, Gross L, Negron VC, Charlesworth C, Debiec H, et al. Protocadherin 7-Associated Membranous Nephropathy. Abstract FR-OR36. ASN (2020).

22. Al-Rabadi L, Caza T, Avillach C, Rodan AR, Williams B, Abraham J, et al. High Temperature Recombinant Protein A1 (HTRA1): A Novel Antigen in Membranous Nephropathy. Abstract FR-OR35. ASN (2020).

23. Sethi S, Madden BJ, Debiec H, Charlesworth MC, Gross LA, Ravindran A, et al. Exostosin 1/exostosin 2 - associated membranous nephropathy. J Am Soc Nephrol. (2019) 30:1123-36. doi: 10.1681/ASN.2018080852

24. Caza T, Hassen S, Kuperman MB, Sharma S, Dvanajscak Z, Arthur J, et al. Neural cell adhesion molecule 1 is a novel autoantigen in membranous lupus nephritis. Kidney Int. (2020). doi: 10.1016/j.kint.2020.09.016. [Epub ahead of print].

25. Ravindran A, Moura MC, Fervenza FC, Nasr SH, Alexander MP, Fidler ME, et al. In patients with membranous lupus nephritis, exostosin-positivity and exostosin-negativity represent two different phenotypes. J Am Soc Nephrol. (2021) 32:695-706. doi: 10.1681/ASN.2020081181

26. Sethi S. New 'antigens' in membranous nephropathy. J Am Soc Nephrol. (2020) 32:268-78. doi: 10.1681/ASN.2020071082

27. Ahmad SB, Appel G. Antigens, antibodies, and membranous nephropathy: a decade of progress. Kidney Int. (2020) 97:2931. doi: 10.1016/j.kint.2019.10.009

28. Larsen CP, Cossey LN, Beck LH. THSD7A staining of membranous glomerulopathy in clinical practice reveals cases with dual autoantibody positivity. Mod Pathol. (2016) 29:421-6. doi: 10.1038/modpathol.2016.32

29. Wang J, Cui Z, Lu J, Probst C, Zhang Y-M, Wang X, et al. Circulating antibodies against thrombospondin type-I domain-containing 7A in Chinese patients with idiopathic membranous nephropathy. Clin J Am Soc Nephrol. (2017) 12:1642-51. doi: 10.2215/CJN.01460217

30. Zaghrini C, Seitz-Polski B, Justino J, Dolla G, Payre C, Jourd-Chiche N, et al. Novel ELISA for thrombospondin type 1 domain-containing 7A autoantibodies in membranous nephropathy. Kidney Int. (2019) 95:66679. doi: 10.1016/j.kint.2018.10.024

31. Tominaga K, Uchida T, Imakiire T, Itoh K, Shimazaki H, Nakanishi K, et al. Anti-neutrophil cytoplasmic antibody-associated glomerulonephritis with detection of myeloperoxidase and phosphlipase A2 receptor in membranous nephropathy-lesions: report of two patients with microscopic polyangiitis. BMC Nephrol. (2018) 19:120. doi: 10.1186/s12882-018-0922-5

32. Alawieh R, Brodsky SV, Satoskar AA, Nadasdy T, Parikh SV, Rovin B, Cassol C. Membranous nephropathy with crescents. Kidney Int Rep. (2020) 5:537-41. doi: 10.1016/j.ekir.2020.01.010
33. Nikolopoulou A, Huang-Doran I, McAdoo SP, Griffith ME, Cook HT, Pusey CD. Membranous nephropathy with crescents. Kidney Int Rep. (2019 4:157784.

34. Debiec H, Ronco P. PLA2R autoantibodies and PLA2R glomerular deposits in membranous nephropathy. N Engl J Med. (2011) 364:68990. doi: 10.1056/NEJMc1011678

35. Svobodova B, Honsova E, Ronco P, Tesar V, Debiec H. Kidney biopsy is a sensitive tool for retrespective diagnosis of PLA2Rrelated membranous nephropathy. Nephrol Dial Transplant. (2013) 28:1839-44. doi: 10.1093/ndt/gfs439

36. Van de Loght A-E, Hofstra JM, Wetzels JFM. Serum anti-PLA2R antibodies can be initially absent in idiopathic membranous nephropathy: seroconversion after prolonged follow-up. Kidney Int. (2015) 87:12634. doi: 10.1038/ki.2015.34

37. Ramachandran R, Kumar V, Nada R, Jha V. Serial monitoring of anti-PLA2R in initial PLA2R-negative patients with primary membranous nephropathy. Kidney Int. (2015) 88:1198-9. doi: 10.1038/ki.2015.310

38. Ryan MS, Satoskar AA, Nadasdy GM, Brodsky SV, Hemminger JA, Nadasdy T. Phospholipase A2 receptor staining is absent in many kidney biopsies with early-stage membranous glomerulonephritis. Kidney Int. (2016) 89:14023. doi: 10.1016/j.kint.2015.12.057

39. Lin L, Wang WM, Pan XX, Xu J, Gao CN, Zhang W, et al. Biomarkers to detect membranous nephropathy in Chinese patients. Oncotarget. (2016) 7:67868-79. doi: 10.18632/oncotarget.12014

40. Couser WG. The pathogenesis of human membranous nephropathy: we are almost there. Kidney Int. (2020) 97:849-52. doi: 10.1016/j.kint.2020. 01.037

41. Ravindran A, Madden B, Charlesworth MC, Sharma R, Sethi A, Debiec $\mathrm{H}$, et al. Proteomic analysis of complement proteins in membranous nephropathy. Kidney Int Rep. (2020) 5:618-26. doi: 10.1016/j.ekir.2020. 01.018

42. Brglez V, Boyer-Suavet S, Seitz-Polski B. Complement pathways in membranous nephropathy: complex and multifactorial. Kidney Int Rep. (2020) 5:572-4. doi: 10.1016/j.ekir.2020.02.1033

43. Tomas NM, Hoxha E, Reinicke AT, Fester L, Helmchen U, Gerth J, et al. Autoantibodies against thrombospondin type 1 domain-containing 7A induce membranous nephropathy. J Clin Invest. (2016) 126:251932. doi: 10.1172/JCI85265

44. Tomas NM, Meyer-Schwesinger C, von Spiegel H, Kotb AM, Zahner G, Hoxha E, et al. A heterologous model of thrombospondin type 1 domaincontaining 7A-associated membranous nephropathy. J Am Soc Nephrol. (2017) 28:3262-77. doi: 10.1681/ASN.2017010030

45. Meyer-Schwesinger C, Tomas NM, Dehde S, Seifert L, Hermans-Borgmeyer I, Wiech T, et al. A novel mouse model of phospholipase A2 receptor 1 associated membranous nephropathy. Clin J Am Soc Nephrol. (2017) 12:98397. doi: 10.1016/j.kint.2019.10.022

46. Cornaby C, Gibbons L, Mayhew V, Sloan CS, Welling A, Poole BD. B cell epitope spreading: mechanisms and contribution to autoimmune diseases. Immunol Lett. (2015) 163:56-68. doi: 10.1016/j.imlet.2014.11.001

47. Seitz-Polski B, Dolla G, Payré C, Girard CA, Polidori J, Zorzi K, et al. Epitope spreading of autoantibody response to PLA2R associates with poor prognosis in membranous nephropathy. J Am Soc Nephrol. (2016) 27:151733. doi: 10.1681/ASN.2014111061

48. Stanescu HC, Arco-Burgos M, Medlar A, Bockenhauer D, Kottgen A, Dragomirescu L, et al. Risk HLA-DQA1 and PLA2R1 alleles in idiopathic membranous nephropathy. N Engl J Med. (2011) 364:61626. doi: 10.1056/NEJMoa1009742

49. Lyons PA, Rayner TF, Trivedi S, Holle JU, Watts RA, Jayne DR, et al. Genetically distinct subsets within ANCA-associated vasculitis. N Engl J Med. (2012) 367:214-23. doi: 10.1056/NEJMoa1108735

50. Lv J, Hou W, Zhou X, Liu G, Zhou F, Zhao N, et al. Interaction between PLA2R1 and HLA-DQA1 variants associates with anti-PLA2R antibodies and membranous nephropathy. J Am Soc Nephrol. (2013) 24:13239. doi: 10.1681/ASN.2012080771

51. Kanigicherla D, Gummadova J, McKenzie EA, Roberts SA, Harris S, Nikam M, et al. Anti-PLA2R antibodies measured by ELISA predict long-term outcome in a prevalent population of patients with idiopathic 
membranous nephropathy. Kidney Int. (2013) 83:940-8. doi: 10.1038/ki.20 12.486

52. Xie J, Liu L, Mladkova N, Li Y, Ren H, Wang W, et al. The genetic architecture of membranous nephropathy and its potential to improve noninvasive diagnosis. Nat Commun. (2020) 11:1600. doi: 10.1038/s41467-02015383-W

53. Kiryluk K, Li Y, Sanna-Cherchi S, Rohanizadegan M, Suzuki H, Eitner F, et al. Geographic differences in genetic susceptibility to IgA nephropathy: GWAS replication study and geospatial risk analysis. PLoS Genet. (2012) 8:e1002765. doi: 10.1371/journal.pgen. 1002765

54. Bobart SA, Fervenza FC. Kidney biopsy is required for nephrotic syndrome with PLA2R+ and normal kidney function: the CON view. Kidney360. (2020) 1:890-3. doi: $10.34067 /$ KID.0003262020

55. Hogan JJ. Kidney biopsy is required for nephrotic syndrome with PLA2R+ and normal kidney function: PRO. Kidney360. (2020) 1:8879. doi: 10.34067/KID.0003752020

56. Burbelo PD, Joshi M, Chaturvedi A, Little DJ, Thurlow JS, Waldman $M$, et al. Detection of PLA2R autoantibodies before the diagnosis of membranous nephropathy. J Am Soc Nephrol. (2020) 31:308-17. doi: 10.1681/ASN.2019050538

57. Wang Q, Dong ZZ, Zhang WG, Liu XM, Qu YL, Duan SW, et al. Diagnostic efficacy of serum anti-phospholipase A2 receptor antibodies for idiopathic membranous nephropathy in patients with diabetic kidney disease. Clin Chim Acta. (2020) 502:222-6. doi: 10.1016/j.cca.2019. 11.004

58. De Vriese AS, Glassock RJ, Nath KA, Sethi S, Fervenza FC. A proposal for a serology-based approach to membranous nephropathy. J Am Soc Nephrol. (2017) 28:421-30. doi: 10.1681/ASN.2016070776

59. Leeaphorn N, Kue-A-Pai P, Thamcharoen N, Ungprasert P, Stoke MB, Knight EL. Prevalence of cancer in membranous nephropathy: a systematic review and meta-analysis of observational studies. Am J Nephrol. (2014) 40:2935. doi: 10.1159/000364782

60. Hoxha E, Wiech T, Stahl PR, Zahner G, Tomas NM, Meyer-Schwesinger C, et al. A mechanism for cancer-associated membranous nephropathy. $\mathrm{N} \mathrm{Engl} \mathrm{J}$ Med. (2016) 374:1995-6. doi: 10.1056/NEJMc1511702

61. Pozdzik A, Brochériou I, David C, Touzani F, Goujon JM, Wissing KM. Membranous nephropathy and anti-podocytes antibodies: Implications for the diagnostic workup and disease management. Biomed Res Int. (2018) 2018:6281054. doi: 10.1155/2018/6281054

62. Zhang C, Zhang M, Chen D, Ren Q, Xu W, Zeng C, et al. Features of phospholipase A2 receptor and thrombospondin type-1 domain-containing 7A in malignancy-associated membranous nephropathy. Clin Pathol. (2019) 72:705-11. doi: 10.1136/jclinpath-2019-205852

63. Radice A, Pieruzzi F, Trezzi B, Ghiggeri G, Napodano P, D’Amico M, et al. Diagnostic specificity of autoantibodies to M-type phospholipase A2 receptor (PLA2R) in differentiating idiopathic membranous nephropathy (IMN) from secondary forms and other glomerular diseases. J Nephrol. (2018) 31:2718. doi: 10.1007/s40620-017-0451-5

64. Von Haxthausen F, Reinhard L, Pinnschmidt HO, Rink M, Soave A, Hoxha E, et al. Antigen-specific IgG subclasses in primary and malignancy-associated membranous nephropathy. Front Immunol. (2018) 9:3035. doi: 10.3389/fimmu.2018.03035

65. Caza T, Hassen S, Dvanajscak Z, Kuperman M, Edmondson R, Herzog $\mathrm{C}$, et al. NELL1 is a target antigen in malignancy-associated membranous nephropathy. Kidney Int. (2020) doi: 10.1016/j.kint.2020.07.039. [Epub ahead of print].

66. Zhang D, Zhang C, Bian F, Zhang W, Jiang G, Zou J. Clinicopathological features in membranous nephropathy with cancer: a retrospective singlecenter study and literature review. Int J Biol Markers. (2019) 34:40613. doi: $10.1177 / 1724600819882698$

67. Hofstra JM, Debiec H, Short CD, Pellé T, Kleta R, Mathieson PW, et al. Antiphospholipase A2 receptor antibody titre and subclass in idiopathic membranous nephropathy. J Am Soc Nephrol. (2012) 23:173543. doi: 10.1681/ASN.2012030242

68. Hofstra JM, Beck LH Jr., Beck DM, Wetzels JF, Salant DJ. Antiphospholipase A2 receptor antibodies correlate with clinical status in idiopathic membranous nephropathy. Clin J Am Soc Nephrol. (2011) 6:128691. doi: $10.2215 /$ CJN.07210810
69. Jullien P, Seitz-Polski B, Maillard N, Thibaudin D, Laurent B, Ollier E, et al. Anti-phospholipase A2 receptor antibody levels at diagnosis predicts spontaneous remission of idiopathic membranous nephropathy. Clin Kidney J. (2017) 10:209-14. doi: 10.1093/ckj/sfw121

70. Timmermans S, Abdul Hamid MA, Cohen Tervaert JW, Damoiseaux J, van Paassen P. Anti-PLA2R antibodies as a prognostic factor in PLA2R-related membranous nephropathy. Am J Nephrol. (2014) 142:2934. doi: 10.1159/000437236

71. Rodas LM, Matas-Garcia A, Barrox X, Blasco M, Vinas O, Llobell A, et al. Antiphospholipase 2 receptor antibody levels to predict complete spontaneous remission in primary membranous nephropathy. Clin Kidney J. (2019) 12:36-41. doi: 10.1093/ckj/sfy005

72. Hoxha E, Thiele I, Zahner G, Panzer U, Harendza S, Stahl RAK. Phospholipase A2 receptor autoantibodies and clinical outcome in patients with primary membranous nephropathy. J Am Soc Nephrol. (2014) 25:135766. doi: 10.1681/ASN.2013040430

73. Seitz-Polski B, Debiec H, Rousseau A, Dahan K, Zaghrini C, Payré C, et al. Phospholipase A2 receptor 1 epitope spreading at baseline predicts reduced lilkelihood of remission in membranous nephropathy. J Am Soc Nephrol. (2018) 29:401-8. doi: 10.1681/ASN.2017070734

74. Cosio FG, Cattran DC. Recent advances in our understanding of recurrent primary glomerulonephritis after kidney transplantation. Kidney Int. (2017) 91:304-14. doi: 10.1016/j.kint.2016.08.030

75. Passerini P, Malvica S, Tripodi F, Cerutti R, Messa P. Membranous nephropathy $(\mathrm{MN})$ recurrence after renal transplantation. Front Immunol. (2019) 10:1326. doi: 10.3389/fimmu.2019.01326

76. Stahl R, Hoxha E, Fechner K. PLA2R autoantibodies and recurrent membranous nephropathy after transplantation. N Engl J Med. (2010) 363:496-8. doi: 10.1056/NEJMc1003066

77. Quintana LF, Blasco M, Seras M, Pérez NS, López-Hoyos M, Villarroel $\mathrm{P}$, et al. Antiphospholipase A2 receptor antibocy levels predict the risk of posttransplantation recurrence of membranous nephropathy. Transplantation. (2015) 99:1709-14. doi: 10.1097/TP.0000000000 000630

78. Gupta G, Fattah H, Ayalon R, Kidd J, Gehr T, Quintana LF, et al. Pre-transplant phospholipase A2 receptor autoantibody concentration is associated with clinically significant recurrence of membranous nephropath post-kidney transplantation. Clin Transplant. (2016) 30:461-9. doi: 10.1111/ctr.12711

79. Hoxha E, Harendza S, Pinnschmidt H, Panzer U, Stahl RAK. M-type phospholipase A2 receptor autoantibodies and renal function in patients with primary membranous nephropathy. Clin J Am Soc Nephrol. (2014) 9:1883-90. doi: 10.2215/CJN.03850414

80. Ruggenenti P, Debiec H, Ruggiero B, Chianca A, Pellé T, Gaspari F, et al. Anti-phospholipase A2 receptor antibody titer predicts post-rituximab outcome of membranous nephropathy. J Am Soc Nephrol. (2015) 26:254558. doi: 10.1681/ASN.2014070640

81. Beck LH Jr., Fervenza FC, Beck DM, Bonegion GB, Malik FA, Erickson SB, et al. Rituximab-induced depletion of anti-PLA2R autoantibodies predicts response in membranous nephropathy. J Am Soc Nephrol. (2011) 22:154350. doi: 10.1681/ASN.2010111125

82. Qin W, Beck LH Jr., Zeng C, Chen Z, Li S, Zuo K, et al. Anti-phospholipase A2 receptor antibody in membranous nephropathy. J Am Soc Nephrol. (2011) 22:1137-43. doi: 10.1681/ASN.2010090967

83. Ramachandran R, Yadav AK, Kumar V, Inamdar N, Nada R, Gupta $\mathrm{KL}$, et al. Temporal association between PLA2R antibodies and clinical outcomes in primary membranous nephropathy. Kidney Int Rep. (2018) 3:142-7. doi: 10.1016/j.ekir.2017.09.001

84. Dahan K, Debiec H, Plaisier E, Cachanado M, Rousseau A, Wakselman $\mathrm{L}$, et al. Rituximab for severe membranous nephropathy: a 6month trial with extended follow-up. J Am Soc Nephrol. (2017) 28:348-58. doi: 10.1681/ASN.2016040449

85. Fervenza FC, Appel GB, Barbour SJ, Rovin BH, Lafayette RA, Aslam N, et al. Rituximab or cyclosporine in the treatment of membranous nephropathy. $N$ Engl J Med. (2019) 381:36-46. doi: 10.1056/NEJMoa1814427

86. Fernández-Juárez G, Rojas-Rivera J, van de Logt AE, Justino J, Sevillano A, Caravaca-Fontán F, et al. The STARMEN trial indicates that alternating treatment with corticosteroids and cyclophosphamide is superior to sequential treatment with tacrolimus and rituximab in primary membranous 
nephropathy. Kidney Int. (2020). doi: 10.1016/j.kint.2020.10.014. [Epub ahead of print].

87. Barrett C, Willcocks LC, Jones RB, Tarzi RM, Henderson RB, Cai G, et al. Effect of belimumab on proteinuria and anti-phospholipase A2 receptor autoantibody in primary membranous nephropathy. Nephrol Dial Transplant. (2020) 35:599-606. doi: 10.1093/ndt/gfz086

88. Seitz-Polski B, Dahan K, Debiec H, Rousseau A, Andreani M, Zaghrini C, et al. High-dose rituximab and early remission in PLA2R1-related membranous nephropathy. Clin J Am Soc Nephrol. (2019) 14:117382. doi: $10.2215 /$ CJN.11791018
Conflict of Interest: The authors declare that the research was conducted in the absence of any commercial or financial relationships that could be construed as a potential conflict of interest.

Copyright (c) 2021 Tesar and Hruskova. This is an open-access article distributed under the terms of the Creative Commons Attribution License (CC BY). The use, distribution or reproduction in other forums is permitted, provided the original author(s) and the copyright owner(s) are credited and that the original publication in this journal is cited, in accordance with accepted academic practice. No use, distribution or reproduction is permitted which does not comply with these terms. 